\title{
Functionalized selenium nanoparticles for targeted delivery of doxorubicin to improve non-small-cell lung cancer therapy
}

This article was published in the following Dove Press journal:

International Journal of Nanomedicine

\section{Yu Xia \\ Yi Chen \\ Liang Hua \\ Mingqi Zhao \\ Tiantian Xu \\ Changbing Wang \\ Yinghua Li \\ Bing Zhu}

Virus Laboratory, Guangzhou Institute of Pediatrics, Guangzhou Women and Children's Medical Center, Guangzhou Medical University, Guangzhou,

People's Republic of China
Correspondence: Bing Zhu

Virus Laboratory, Guangzhou Institute of Pediatrics, Guangzhou Women and Children's Medical Center, Guangzhou Medical University, No 318 Renminzhong Road, Yuexiu District, Guangzhou, Guangdong 510120, People's Republic of China

Tel +862081322725

Email zhubing2017@hotmail.com
Background: Selenium nanoparticles (SeNPs) loaded with chemotherapeutic drugs provided a novel perspective for cancer therapy.

Materials and methods: Here, SeNPs were modified with cyclic peptide (Arg-Gly-AspD-Phe-Cys [RGDfC]) to fabricate tumor-targeting delivery carrier RGDfC-SeNPs and, then, doxorubicin (DOX) was loaded to the surface of RGDfC-SeNPs for improving the antitumor efficacy of DOX in non-small-cell lung carcinoma therapy.

Results: The chemical structure characterization of RGDfC-Se@DOX showed that DOX was successfully loaded to the surface of RGDfC-SeNPs to prepare functionalized antitumor drug delivery system RGDfC-Se@DOX. RGDfC-Se@DOX exhibited effective cellular uptake in A549 cells and entered A549 cells mainly by clathrin-mediated endocytosis pathway. Compared to free DOX or Se@DOX at the equivalent dose of DOX, RGDfC-Se@DOX showed greater activity to inhibit A549 cells' proliferation and migration/invasion and induce A549 cells' apoptosis. More importantly, compared with passive targeting delivery system $\mathrm{Se} @ \mathrm{DOX}$, active targeting delivery system RGDfC-Se@DOX exhibited more significant antitumor efficacy in vivo.

Conclusion: Taken together, RGDfC-Se@DOX may be a novel promising drug candidate for the lung carcinoma therapy.

Keywords: nanoscale drug carrier, antitumor, chemotherapy, RGDfC peptide, apoptosis

\section{Introduction}

Lung cancer that is known as pulmonary carcinoma or carcinoma of the lung can be categorized into non-small-cell lung cancer (NSCLC) and small-cell lung cancer (SCLC). ${ }^{1-3}$ The NSCLC makes up approximately $80 \%$ of all kinds of lung cancer, and the incidence and mortality ratio has been quickly rising worldwide. ${ }^{4,5}$ Chemotherapy is still an important treatment strategy for cancer therapy clinically. Doxorubicin (DOX) is a very common anticancer drug clinically. ${ }^{6,7}$ Nevertheless, the anticancer efficacy of DOX is not so ideal as expected partly because of its lack of targeted specificity, poor solubility, inadequate drug accumulation in the tumor, and serious side effects. ${ }^{8,9}$ Therefore, it is very crucial to improve the efficiency of chemotherapy by using new technologies.

Recently, nanotechnology application in the field of cancer therapy has provided a large number of advantages, such as early diagnosis, multifunctional therapy, and drug delivery systems. ${ }^{10,11}$ Many nanoscale drug delivery systems including polymers, mesoporous silica, liposomes, and nanoparticles were used to fabricate the nanoscale anticancer drug delivery systems with passive tumor-targeting property, which resulted from enhanced permeability and retention (EPR) effects. ${ }^{12-14}$ In these nanoscale drug delivery systems, selenium nanoparticles (SeNPs) have received a lot of attention as 
drug carriers. ${ }^{15,16}$ First, selenium (Se) as a trace element is very important to human biological process and involves many physiological functions. ${ }^{17,18}$ Second, Se plays a key role in cancer prevention and immune response. ${ }^{19}$ Moreover, SeNPs showed some other advantages as drugs' carrier, for example, the controlled size, potent drug loading capacity, improved antitumor effect, and low cytotoxicity. ${ }^{20,21}$ Thus, SeNPs gradually developed into a excellent anticancer drug carrier. However, there is still some deficiency, especially the lack of active tumor-targeted capacity still existed in such delivery carrier. ${ }^{22}$ To obtain high targeting ability, several tumor-targeted molecules, such as arg-gly-asp (RGD) peptide, hyaluronic acid, and folate, were used for decorating the nanoparticles. ${ }^{23-26}$

The NSCLC A549 cell was a typical lung cancer cell; thus, A549 cells were used for lung cancer therapy researches in this study. In order to improve the anticancer capacity of DOX in NSCLC A549 cells, cyclic peptide (Arg-Gly-AspD-Phe-Cys [RGDfC]) was loaded to the surface of SeNPs. The RGDfC peptide is able to specifically bind with $\alpha_{v \beta 3}$ integrins, which are overexpressed on various cancer cells. ${ }^{27}$ RGDfC was loaded to the surface of SeNPs to fabricate the tumor-targeted carrier RGDfC-SeNPs, and DOX was loaded to the surface of RGDfC-SeNPs to prepare functionalized antitumor nanomedicine RGDfC-Se@DOX, which was effectively capable of inhibiting the proliferation, migration, and invasion of the A549 cells and obtaining favorable in vivo antitumor efficacy.

\section{Materials and methods Materials}

Cyclic peptide (RGDfC) was purchased from China Peptides Co., Ltd. (Shanghai, China) DOX hydrochloride (DOX·HCl), sodium selenite $\left(\mathrm{Na}_{2} \mathrm{SeO}_{3}\right)$, ascorbic acid (vitamin $\mathrm{C}[\mathrm{Vc}]$ ), dimethyl sulfoxide (DMSO), Annexin V-fluorescein isothiocyanate (FITC)/PI kit, DAPI and MTT were purchased from Sigma-Aldrich Co. (St Louis, MO, USA). The antibody was obtained from Cell Signaling Technology (Danvers, MA, USA). Penicillin-streptomycin, DMEM, and FBS were obtained from Thermo Fisher Scientific (Waltham, MA, USA).

\section{Preparation and characterization of RGDfC-Se@DOX}

SeNPs were prepared as previously reported with partial modification. ${ }^{28}$ Briefly, $0.25 \mathrm{~mL}$ of $\mathrm{Na}_{2} \mathrm{SeO}_{3}(0.1 \mathrm{M})$ solution and $2 \mathrm{~mL}$ of $\mathrm{Vc}(0.5 \mathrm{mM})$ solution were slowly added into $22.75 \mathrm{~mL}$ of Milli-Q water in a $50 \mathrm{~mL}$ beaker. Then, the solution mixtures were magnetically stirred for $30 \mathrm{~min}$ at room temperature to prepare SeNPs. Then, RGDfC was added to SeNPs and was magnetically stirred for $2 \mathrm{~h}$ at room temperature to prepare RGDfC-modified SeNPs (RGDfCSeNPs). After that, $2 \mathrm{mg}$ of DOX $\mathrm{HCl}$ was dissolved in $5 \mu \mathrm{L}$ of DMSO and, then, added into RGDfC-SeNPs, and the mixed solutions were magnetically stirred for another 8 h. At last, high-purity RGDfC-Se@DOX was obtained via dialyzing reaction solutions for $4 \mathrm{~h}$. Morphology of nanoparticles was characterized by transmission electron microscopy (TEM). The average size of nanoparticles was characterized by dynamic light scattering (DLS) analysis. The elemental analysis of nanoparticles was characterized by energy-dispersive X-ray (EDX). The chemical structures were characterized by Fourier transform infrared (FTIR). The size of nanoparticles in the water solution was continuously observed for 16 days.

\section{Cell culture}

NSCLC A549 cells and human umbilical vein endothelial cells (HUVEC) were purchased from American Type Culture Collection (ATCC) (Manassas, VA, USA) and were cultivated in DMEM with $10 \% \mathrm{FBS}$ at $37^{\circ} \mathrm{C}$ with $5 \% \mathrm{CO}_{2}$.

\section{Cellular uptake study}

A549 cells at the density of $4 \times 10^{4}$ cells/well were incubated in a 24-well plate for $12 \mathrm{~h}$. Then, A549 cells were exposed to Se@DOX or RGDfC-Se@DOX at the DOX dose of $4 \mu \mathrm{g} / \mathrm{mL}$ for 1, 2, and $4 \mathrm{~h}$. Then, the cells were washed with PBS and stained with DAPI for $15 \mathrm{~min}$. Subsequently, the cells were washed with PBS three times and the images of cells were observed under the fluorescence microscope (DMi8; Leica Microsystems, Wetzlar, Germany).

A549 cells were used to research the cellular uptake mechanism. Briefly, $0.5 \mathrm{~mL}$ of A549 cell suspension at a density of $2 \times 10^{5}$ cells $/ \mathrm{mL}$ was seeded in a 12-well plate and incubated at $37^{\circ} \mathrm{C}$ for $24 \mathrm{~h}$. After that, the cells were exposed to RGDfC-Se@DOX a the DOX dose of $4 \mu \mathrm{g} / \mathrm{mL}$ for $4 \mathrm{~h}$ in the absence of inhibitor at $4^{\circ} \mathrm{C}$, or with $3 \mathrm{mg} / \mathrm{mL}$ of sodium azide $\left(\mathrm{NaN}_{3}\right)+50$ mM 2-deoxy-D-glucose (DOG) or various cellular uptake inhibitors, such as chlorpromazine $(2 \mu \mathrm{g} / \mathrm{mL})$, amiloride $(5 \mu \mathrm{g} / \mathrm{mL})$, and nystatin $(4 \mu \mathrm{g} / \mathrm{mL})$ at $37^{\circ} \mathrm{C}$. Then, the cells were washed with PBS and gently collected. The collected cells were filtered using $22 \mu \mathrm{m}$ nylon mesh and then measured by flow cytometer (BD Biosciences, San Jose, CA, USA).

\section{In vitro release of DOX}

The release profiles of DOX from RGDfC-Se@DOX were evaluated using the dialysis method. Briefly, $1 \mathrm{mg}$ of 
RGDfC-Se@DOX was suspended in $1 \mathrm{~mL}$ of PBS and transferred into a dialysis tube. Afterward, the tubes were immersed in $49 \mathrm{~mL}$ of buffer solution at $\mathrm{pH} 5.4$ or 7.4 under horizontal shaking (100 rpm). The released DOX was monitored for $30 \mathrm{~h}$ by measuring the fluorescence intensity of DOX at an excitation wavelength of $535 \mathrm{~nm}$. At predetermined time points, aliquots of $1 \mathrm{~mL}$ were removed for fluorescence test and replenished with the equivalent volume of fresh PBS. The amount of payload released was determined by comparing with an experimentally determined standard curve. All the payload release assays were carried out in triplicate, and the results were expressed as the average data with SD.

\section{MTT assay}

The MTT assays were performed to test the cellular cytotoxicity of nanoparticles. ${ }^{29}$ A549 cells at a density of $5 \times 10^{4}$ cells $/ \mathrm{mL}$ were added to a 96 -well culture plate and incubated for $24 \mathrm{~h}$ at $37^{\circ} \mathrm{C}$. The cells were then incubated with free DOX, Se@DOX, and RGDfC-Se@DOX (various equivalent DOX concentrations) or with various concentrations of RGDfC-SeNPs at $37^{\circ} \mathrm{C}$ for $48 \mathrm{~h}$, respectively. Then, the medium was taken away and $100 \mu \mathrm{L}$ of medium containing $20 \mu \mathrm{L}$ of MTT $(0.5 \mathrm{mg} / \mathrm{mL})$ was gently added to each well, followed by incubation for another $4 \mathrm{~h}$. Then, the medium was taken away and $200 \mu \mathrm{L}$ of DMSO was added to each well. The culture plate was incubated for another $0.5 \mathrm{~h}$. The absorbance of each well was tested at $570 \mathrm{~nm}$ by a microplate reader (Bio-Rad Laboratories Inc., Hercules, CA, USA). The cytotoxicity of RGDfC-Se@DOX against HUVEC was also tested using the abovementioned method.

\section{Wound healing assay}

The cell migration was determined by wound healing assays. ${ }^{30}$ In brief, A549 cells $\left(5 \times 10^{4}\right.$ cells/well) were seeded in a 24-well plate and cultured in a complete medium to reach full confluence. The monolayer cells were scratched using the sterile $10 \mu \mathrm{L}$ pipette tip. Then, the medium was taken away, and a fresh DMEM containing 2\% FBS was added to each well. Afterward, A549 cells were co-cultured with DOX, Se@DOX, and RGDfC-Se@DOX at an equivalent DOX concentration of $4 \mu \mathrm{g} / \mathrm{mL}$ and incubated at $37^{\circ} \mathrm{C}$ for $24 \mathrm{~h}$. The scratch closure of scratched monolayer cells was observed and photographed at 0 and $12 \mathrm{~h}$. Average scratched width between the sides of the wound was measured at three random areas. The migration rate was calculated according to the following equation: cell motilities $(\%)=[1-($ distance of scratched area at $24 \mathrm{~h} /$ distance of scratched area at $0 \mathrm{~h})] \times 100 \%$.

\section{Transwell assay}

The cell invasion was analyzed using transwell chambers $(8 \mu \mathrm{m}) .^{31}$ In brief, A549 cells $\left(1 \times 10^{5}\right.$ cells $\left./ \mathrm{mL}\right)$ were seeded into the upper chambers of 24-well transwell plates. Then, the cells were exposed to free DOX, Se@DOX, and RGDfC-Se@DOX at an equivalent DOX concentration of $4 \mu \mathrm{g} / \mathrm{mL}$ for $24 \mathrm{~h}$, respectively. After that, the cells at a density of $5 \times 10^{4}$ cells/well were resuspended in $100 \mu \mathrm{L}$ of serum-free medium and then added to the upper chamber, and subsequently, $300 \mu \mathrm{L}$ of complete medium was added to the bottom chamber. After $24 \mathrm{~h}$, the filter was taken away from the plate and the cells remaining on the upper filter were wiped gently. The cells that migrated to the bottom chamber were fixed using methanol and stained with crystal violet for $2 \mathrm{~min}$. The average migrating cells in six independent views were photographed using microscope.

\section{Flow cytometry assay}

The cell cycle distribution was tested using flow cytometry (BD Biosciences). Briefly, A549 cells were exposed to free DOX, Se@DOX, and RGDfC-Se@DOX at an equivalent DOX concentration of $4 \mu \mathrm{g} / \mathrm{mL}$ for $24 \mathrm{~h}$ and washed twice with cold PBS. The precooled $75 \%$ ethanol was added to the collected cells for fixation at $-20^{\circ} \mathrm{C}$ overnight and then stained with propidium iodine (PI) in the dark place for $30 \mathrm{~min}$. The DNA contents were analyzed by the Modfit software (Verity Software House, Brewer, ME, USA). Flow cytometry was also utilized to examine the effect of RGDfC-Se@DOX on the cellular apoptosis of A549 cells. Briefly, A549 cells were exposed to free DOX or Se@DOX or RGDfC-Se@DOX at an equivalent DOX concentration of $4 \mu \mathrm{g} / \mathrm{mL}$ and washed twice with PBS. Then, the cells were collected and stained with Annexin V-FITC/PI for $30 \mathrm{~min}$ in the dark. Finally, the stained cells were examined by flow cytometry and the data were analyzed by the FlowJo software (Treestar, Ashland, OR, USA).

\section{Xenograft mouse model}

All animal experiments were carried out according to the guideline of Experimental Animal Center of Guangzhou Medical University and approved by the ethics committee of Guangzhou Medical University. The BALB/c nude mice (about 5 weeks old) were used to study the in vivo antitumor efficacy of RGDfC-Se@DOX. A549 cells $\left(1 \times 10^{7}\right.$ cells $\left./ 150 \mu \mathrm{L}\right)$ were injected into the abdomens of mice subcutaneously. The mice were randomly categorized into four groups $(n=6)$ after the volumes of tumors reached $\sim 100 \mathrm{~mm}^{3}$. Subsequently, saline (control group), DOX, Se@DOX, and RGDfC-Se@DOX (at an equivalent 
DOX of $2 \mathrm{mg} / \mathrm{kg}$ ) were intravenously injected to the tumorbearing mice once every other day. The overall treatment time was 21 days. The tumor volumes were reckoned up using the following formula: the tumor volumes $\left(\mathrm{mm}^{3}\right)=1 / 2 \times$ length $\times$ width $^{2}$.

\section{Statistical analysis}

All the data are represented as mean \pm SD. The differences between two groups were compared using one-way ANOVA. The differences were considered to be significant and highly significant at $P<0.05$ and $P<0.01$, respectively.

\section{Results and discussion}

\section{Preparation and characterizations of RGDfC-Se@DOX}

In this study, a novel tumor-targeting delivery system RGDfC-Se@DOX was prepared. The tumor-targeting molecular RGDfC was conjugated with SeNPs to fabricate tumor-targeting delivery carrier RGDfC-SeNPs, and then, the antitumor drug DOX was loaded to the surface of
RGDfC-SeNPs to prepare the tumor-targeting delivery system RGDfC-Se@DOX. As shown in Figure 1A, the average size of RGDfC-Se@DOX was 18 nm. The morphology of nanoparticles in TEM image presented spherical particles with a size from about 7 to $12 \mathrm{~nm}$ (Figure 1B). A strong signal of Se atom, a typical $\mathrm{S}$ atom signal from RGDfC, and a $\mathrm{Cl}$ atom signal from $\mathrm{DOX} \cdot \mathrm{HCl}$ were the elemental compositions of RGDfC-Se@DOX (Figure 1C), indicating that RGDfC and DOX were successfully loaded to the surface of SeNPs. The size of RGDfC-Se@DOX in Figure 1D revealed that RGDfC-Se@DOX kept small sizes $(<100 \mathrm{~nm})$ for 16 days. It is well known that nanoparticles $<200 \mathrm{~nm}$ possess passive targeting ability in solid tumors through the EPR effect. Thus, the relative small size in such nanoparticles can be beneficial to the delivery of drugs to the tumor sites. The FTIR spectra of RGDfC-Se@DOX, DOX, SeNPs, and RGDfC are shown in Figure 2, and the typical peak of SeNPs also appeared in the spectrum of RGDfC-Se@, DOX. The peaks at 1,669 and 1,542/cm correspond to the amide band of RGDfC. After loading RGDfC to SeNPs, the
A

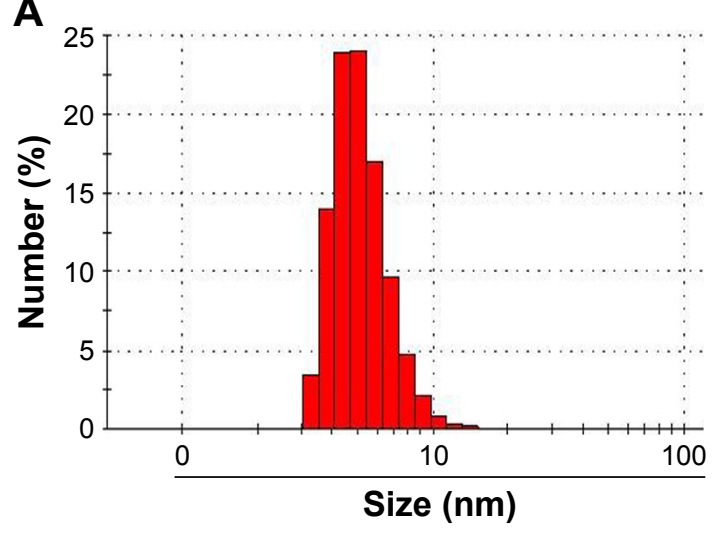

B

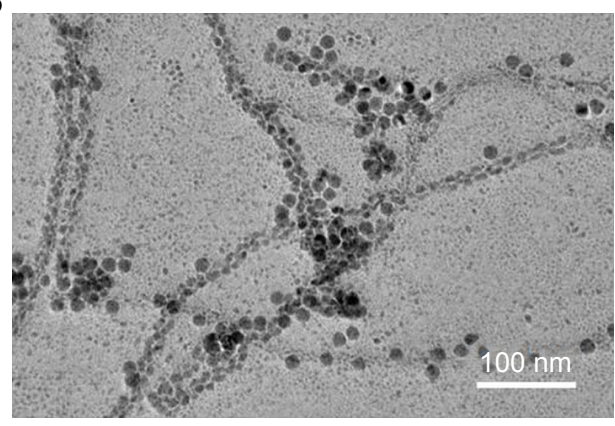

C

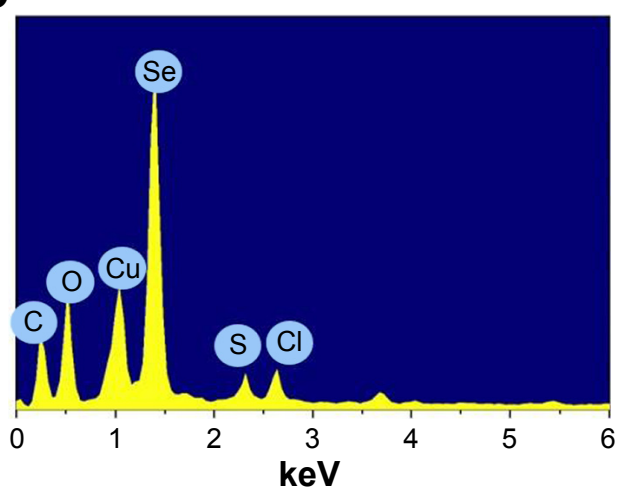

D

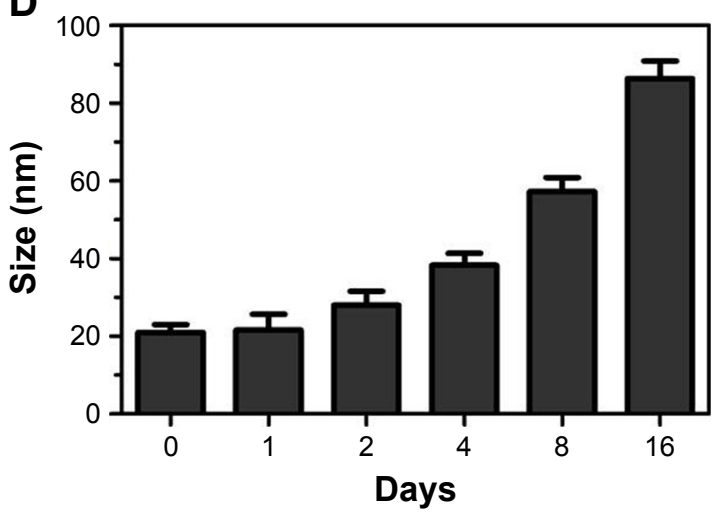

Figure I Characterization of RGDfC-Se@DOX.

Notes: (A) Particle size distribution of RGDfC-Se@DOX. (B) Representative TEM image featuring RGDfC-Se@DOX. (C) EDX analysis of RGDfC-Se@DOX. (D) Stability observation of RGDfC-Se@DOX in aqueous solution. RGDfC-Se@DOX, selenium nanoparticles conjugated with RGDfC and DOX.

Abbreviations: DOX, doxorubicin; EDX, energy-dispersive X-ray; TEM, transmission electron microscopy. 


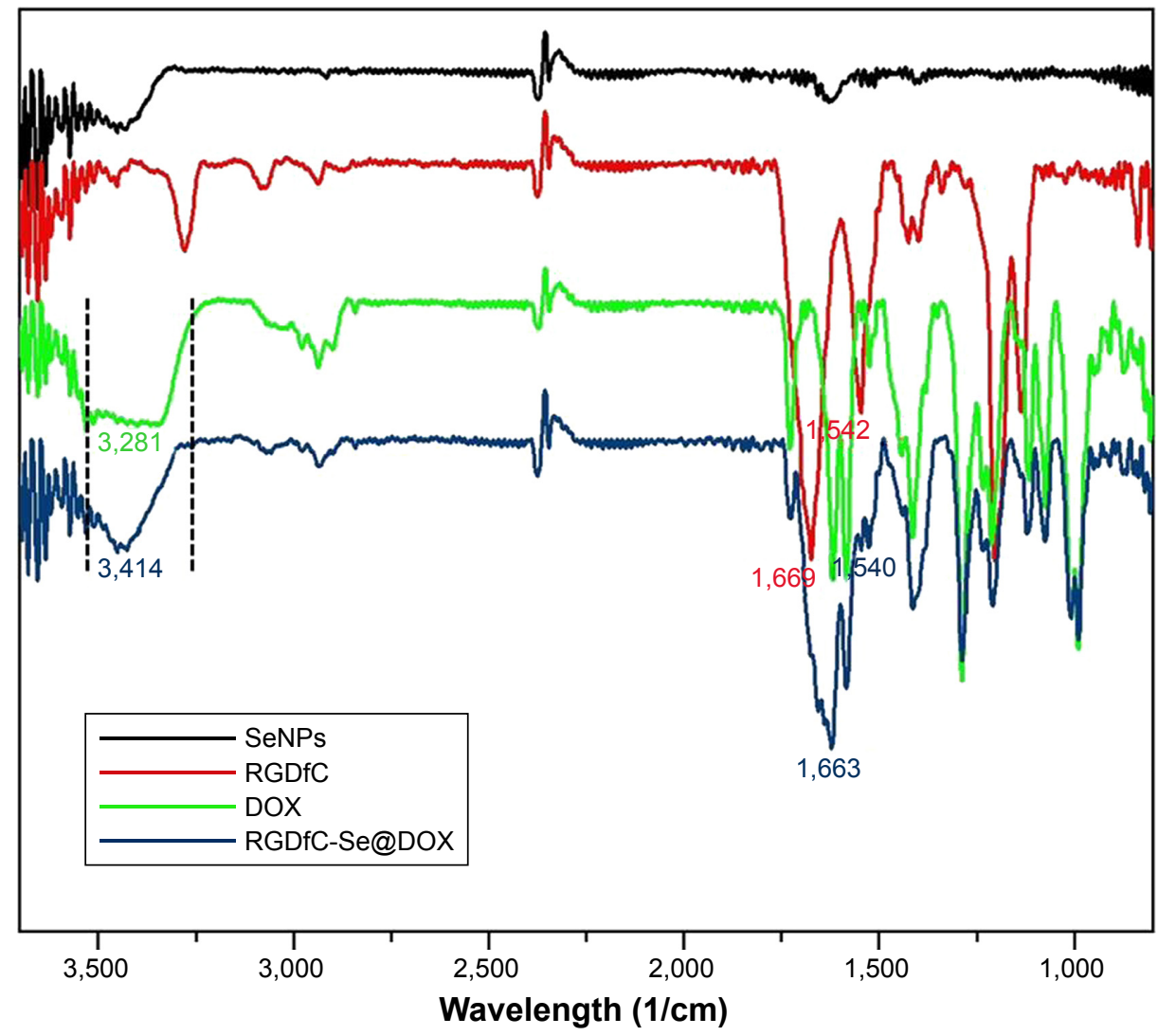

Figure 2 FTIR spectra of SeNPs, RGDfC, DOX, and RGDfC-Se@DOX.

Note: RGDfC-Se@DOX, SeNPs conjugated with RGDfC and DOX.

Abbreviations: DOX, doxorubicin; FTIR, Fourier transform infrared; RGDfC, Arg-Gly-Asp-D-Phe-Cys; SeNPs, selenium nanoparticles.

typical amide bands $\left(1,663\right.$ and $\left.1,540 \mathrm{~cm}^{-1}\right)$ appeared in the spectrum of RGDfC-Se@DOX, indicating the successful linking of SeNPs with RGDfC via amide bonds. The peak at $\sim 3,281 \mathrm{~cm}^{-1}$ assigned to the characteristic hydroxyl group of DOX existed in the spectrum of RGDfC-Se@DOX, verifying the effective linking between DOX and RGDfCSeNPs. The above data showed that RGDfC-Se@DOX was successfully synthesized and exhibited good stability in the water solution.

\section{Cellular uptake studies}

The drug delivery efficiency is closely related to the cellular uptake. High cellular uptake of drug can result in the effective treatment efficacy. Herein, the cellular uptake of passive tumor-targeting nanoparticles Se@DOX and active tumor-targeting nanoparticles RGDfC-Se@DOX was observed in A549 cells under the fluorescence microscope. The red fluorescence from DOX was observed in A549 cells exposed to Se@DOX or RGDfC-Se@DOX for 1 h (Figure 3), suggesting that such nanoparticles could enter the A549 cells irrespective of the active targeting moiety. The intensity of fluorescence in both Se@DOX- and RGDfC-Se@DOXtreated groups increased as the incubation time prolonged to 4 h. Interestingly, the exposure of A549 cells to RGDfC-Se@ DOX resulted in greater cellular uptake compared with Se@DOX, presumably because of RGDfC-mediated specific endocytosis. After the exposure of RGDfC-Se@DOX to A549 cells for 4 h, a small part of RGDfC-Se@DOX obviously entered the nucleus, where RGDfC-Se@DOX exhibited antitumor activity. However, Se@DOX internalized into the cells and wrapped the cytoplasm at the same time of incubation.

It has been reported that nanoparticles could enter the cells in an energy-dependent endocytosis way. ${ }^{32}$ The incubation of A549 cells at $4^{\circ} \mathrm{C}$ or pretreated with $\mathrm{NaN}_{3} / \mathrm{DOG}$ markedly reduced the cellular uptake of nanoparticles (Figure 4), indicating that the endocytosis of RGDfC-Se@DOX is an active energy-dependent process. The cells' endocytosis mainly includes three pathways: clathrin-mediated endocytosis, caveolae-mediated endocytosis, and macropinocytosis. To examine the endocytosis mechanism of RGDfC-Se@DOX in A549 cells, different endocytosis inhibitors were used to 

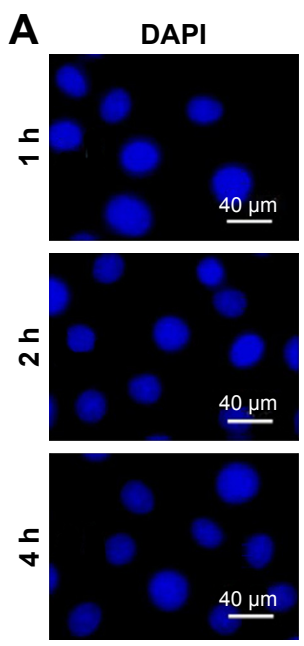

Se@DOX
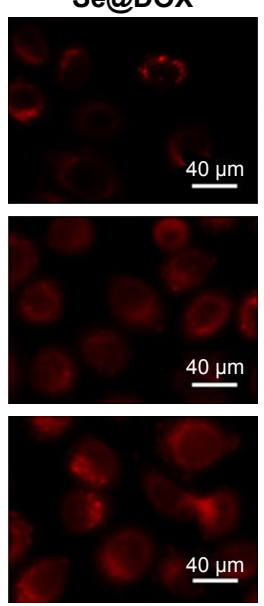

Merged
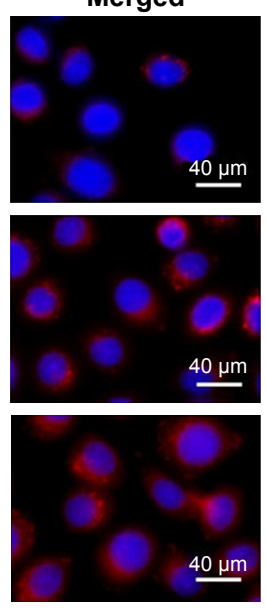

B
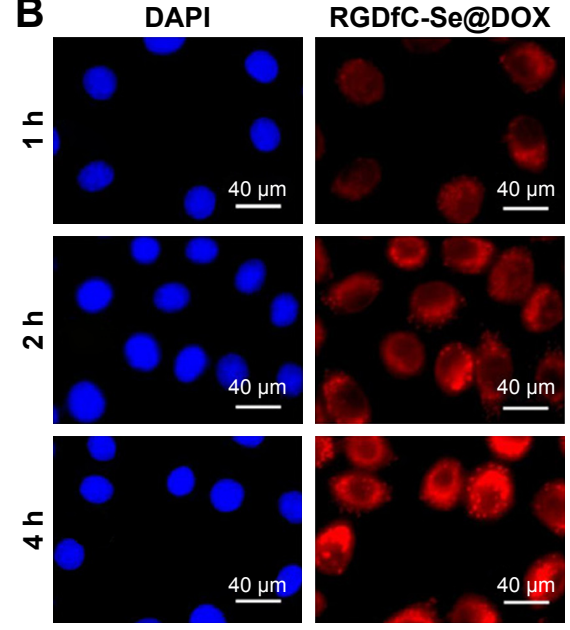

Merged
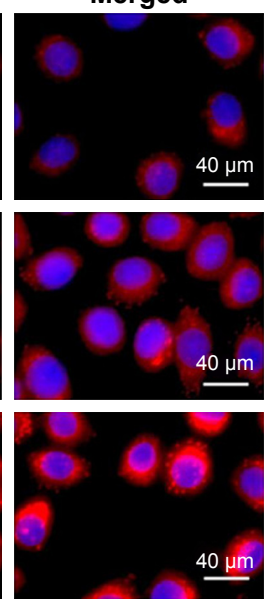

Figure 3 Cellular uptake of Se@DOX (A) and RGDfC-Se@DOX (B) was visualized by red fluorescence from DOX in A549 cells.

Notes: RGDFC-Se@DOX, selenium nanoparticles conjugated with RGDfC and DOX; Se@DOX, selenium nanoparticles conjugated with DOX.

Abbreviations: DOX, doxorubicin; RGDfC, Arg-Gly-Asp-D-Phe-Cys.

study the effect of RGDfC-Se@DOX on cellular uptake. Amiloride, nystatin, and chlorpromazine are usually used to inhibit micropinocytosis, caveolae-mediated endocytosis, and clathrin-associated endocytosis, respectively. After pretreating with amiloride or nystatin, the cellular uptake of RGDfC-Se@DOX was decreased by $32.4 \%$ or $28.3 \%$, respectively. Nevertheless, pretreatment with chlorpromazine resulted in about $49.7 \%$ decrease in the cellular uptake of RGDfC-Se@DOX, suggesting that clathrin-associated endocytosis mainly contributed to the internalization of RGDfC-Se@DOX.

\section{In vitro release of DOX}

To evaluate the in vitro release behavior of RGDfC-Se@ DOX, we investigated the release profiles in two kinds of

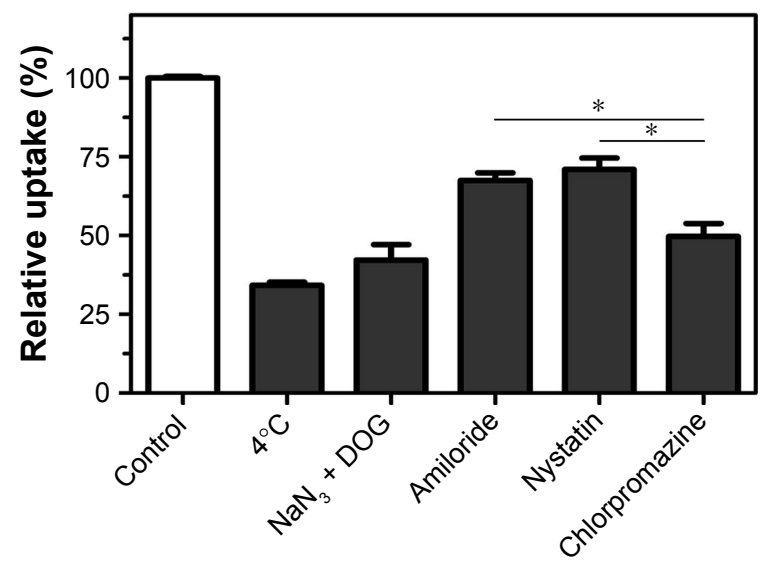

Figure 4 Effects of endocytosis inhibitors and temperature on the internalization of RGDFC-Se@DOX.

Notes: $* p<0.05$ vs chlorpromazine group. RGDfC-Se@DOX, selenium nanoparticles conjugated with RGDFC and DOX.

Abbreviations: DOG, 2-deoxy-D-glucose; DOX, doxorubicin; $\mathrm{NaN}_{3}$, sodium azide; RGDfC, Arg-Gly-Asp-D-Phe-Cys. buffer solutions ( $\mathrm{pH} 5.4$ or 7.4 ) at $37^{\circ} \mathrm{C}$ using the dialysis method. The two different $\mathrm{pH}$ values were used to simulate the normal physiological environment and the endosomal/ lysosomal microenvironments of cancer cells, which are more acidic ( $\mathrm{pH} 4-6)$. The typical release profiles of DOX from RGDfC-Se@DOX are shown in Figure 5A, and the data revealed that its accumulative release behavior was acid dependent, and there was a significant initial burst payload release during the initial $4 \mathrm{~h}$. The release rates of DOX significantly increased when the $\mathrm{pH}$ values changed from 7.4 to 5.4. As shown in Figure 5A, at pH 5.4, 84.2\% of DOX was released in a sustained manner during $30 \mathrm{~h}$. However, at the physiological $\mathrm{pH}$ value ( $\mathrm{pH} 7.4$ ), DOX was released at a slower rate $(57.3 \%)$. The faster release in acidic environment may be due to the decrease in the surface negative charge of SeNPs in acidic $\mathrm{pH}$, which weakens the electrostatic attraction of DOX and facilitates the release of DOX from SeNPs. This $\mathrm{pH}$-dependent DOX release property of RGDfC-Se@DOX is quite beneficial for drug delivery systems in cancer therapy, because most tumors present a more acidic condition than normal tissues. Thus, RGDfC-SeNPs have great potential in carrying and releasing therapeutics for treating cancers.

\section{In vitro cytotoxicity study}

The MTT assay was used to investigate the cytotoxicity of different formulations of DOX against A549 cells in vitro. Free DOX and passive targeting nanoparticle Se@DOX were set as the negative control. As shown in Figure 5, viability of A549 cells exposed to various formulations of DOX gradually declined with increasing DOX concentrations. Free DOX, Se@DOX, and RGDfC-Se@DOX at the DOX 


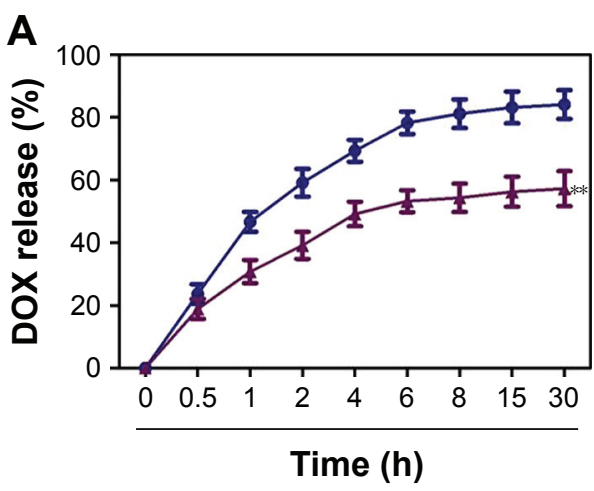

$-\mathrm{pH} 5.4 \leadsto \mathrm{pH} 7.4$
B

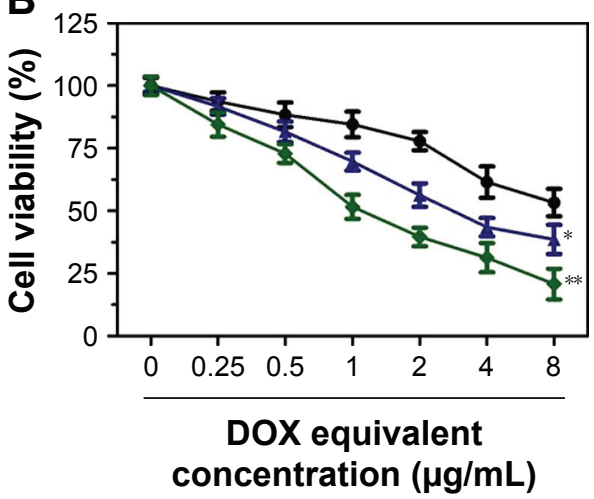

$\bullet$ DOX $\rightarrow$ Se@DOX $\leadsto$ RGDfC-Se@DOX

Figure 5 (A) In vitro pH-triggered release of DOX from RGDfC-Se@DOX.**P<0.0I vs the pH 5.4 group. (B) In vitro cytotoxicity of DOX, Se@DOX, and RGDfC-Se@ DOX on A549 cells. $* P<0.05$ and $* * P<0.01$ vs the free DOX group. RGDfC-Se@DOX, selenium nanoparticles conjugated with RGDfC and DOX; Se@DOX, selenium nanoparticles conjugated with DOX.

Abbreviations: DOX, doxorubicin; RGDfC, Arg-Gly-Asp-D-Phe-Cys.

concentration of $8 \mu \mathrm{g} / \mathrm{mL}$ obviously suppressed A549 cell proliferation, and the cell viability rates were $53.3 \%, 38.6 \%$, and $20.8 \%$, respectively, suggesting that RGDfC-Se@DOX exhibited greater cytotoxicity in A549 cells compared with free DOX and Se@DOX, perhaps because of enhanced cellular uptake of RGDfC-Se@DOX in A549 cells. The cell viability of A549 cells was significantly $<50 \%$ after treatment with RGDfC-Se@DOX at an equivalent DOX dose of $4 \mu \mathrm{g} / \mathrm{mL}$; thus, such dose was used for further biological research. The MTT result indicated that the delivery of DOX using active tumor-targeted carrier RGDfC-SeNPs could effectively enhance the anticancer activity of DOX. The inhibition of proliferation of A549 cells treated with drug carrier RGDfC-SeNPs at the used dose was not obvious (Figure S1), indicating the low cytotoxicity of RGDfCSeNPs. HUVEC were used to evaluate the potential side effects of RGDfC-Se@DOX. As shown in Figure S2, the cytotoxicity of RGDfC-Se@DOX against HUVEC was much lower in comparison with A549 cells, which verified the relatively low cytotoxicity of RGDfC-Se@DOX in human normal cell HUVEC.

\section{RGDfC-Se@DOX suppresses the migration and invasion of A549 cells}

Tumor metastasis and invasion remain the leading causes of death in patients suffering from lung cancer. Effective inhibition of tumor metastasis and invasion is seen as a promising way of delaying tumor growth. ${ }^{33}$ The wound healing assay of the cells was utilized to assess whether RGDfC-Se@ DOX could effectively inhibit the migration of cancer cells. As shown in Figure 6A, wound healing assay results showed that RGDfC-Se@DOX obviously decreased the migration of A549 cells during a $24 \mathrm{~h}$ interval. Meanwhile, the invasion of A549 cells was also strongly inhibited by RGDfC-Se@, DOX (Figure 6C). Furthermore, RGDfC-Se@DOX exhibited higher activity to inhibit A549 cells' migration and invasion in comparison with the free DOX or Se@DOX (Figure 6B and D), indicating that RGDfC-Se@DOX is significantly superior to the free DOX and passive targeting delivery system Se@DOX to inhibit the motility and migration of A549 cells.

\section{Flow cytometry studies}

DOX is a very effective antitumor drug and can induce cancer cell apoptosis. Thus, flow cytometry was used to test whether RGDfC-Se@DOX exhibited greater activity to induce A549 cells' apoptosis compared with free DOX or Se@DOX at the equivalent DOX dose. In this study, the apoptotic cells with DNA fragmentation were reflected as Sub-G1 peaks. Figure 7A shows that Sub-G1 apoptosis peak of cells in the RGDfC-Se@DOX-treated group was stronger (25.72\%) than that in the DOX-treated (12.93\%) and Se@DOX-treatment groups (17.98\%), indicating that RGDfC-Se@DOX exhibited stronger capacity to induce A549 cells' apoptosis at the equivalent DOX dose. However, there is no obvious difference in cell cycle distribution among the different treatment groups.

To further detect the apoptosis of A549 cells treated with various formulations of DOX, the cells were analyzed using Annexin V-FITC/PI dual staining. As shown in Figure 7B, RGDfC-Se@DOX treatment obviously induced A549 cells' apoptosis and resulted in higher cell apoptosis 
A
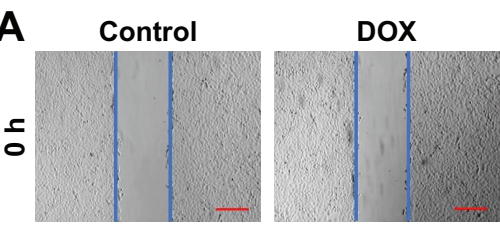

Se@Dox

RGDfC-Se@DoX

\section{$B$}
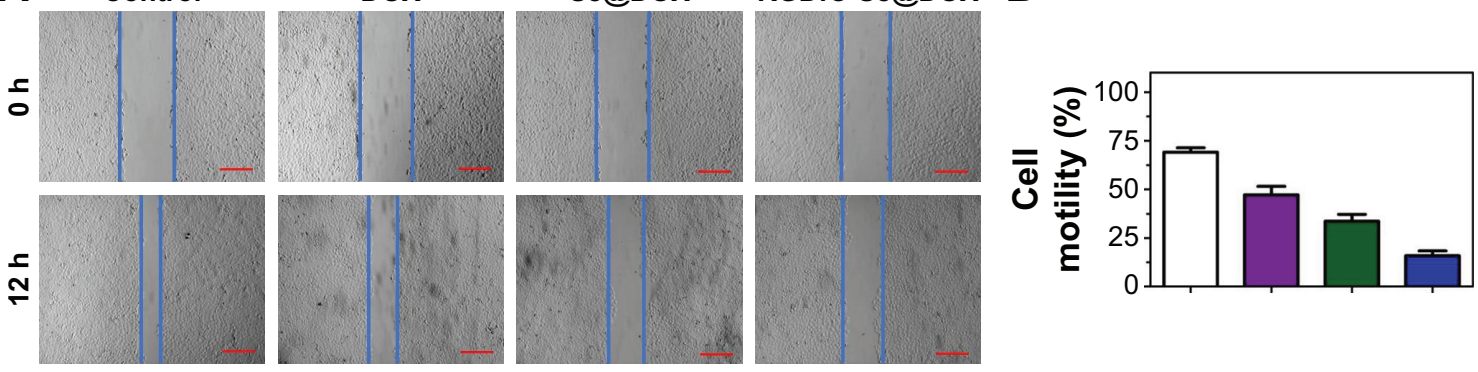

C

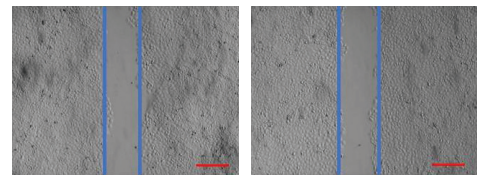

DOX

Se@DOX
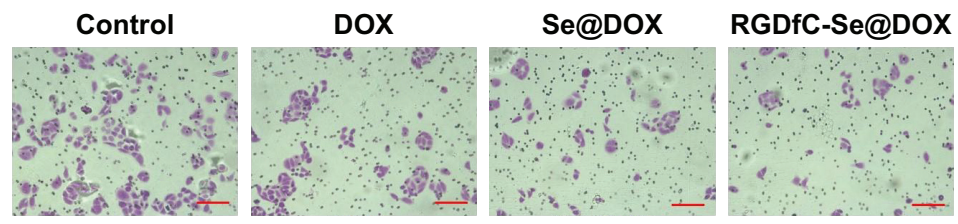

D

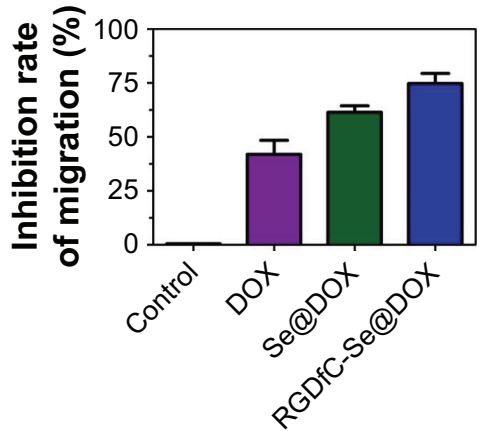

Figure 6 (A) The scratching width of A549 cells was observed at $12 \mathrm{~h}$ after the treatment with DOX, Se@DOX, and RGDfC-Se@DOX. The scale bars are $200 \mu \mathrm{m}$. (B) The cell motility of control, DOX, Se@DOX, and RGDfC-Se@DOX was quantitatively analyzed. (C) The effect of DOX, Se@DOX, and RGDfC-Se@DOX on the migration of A549 cells. The scale bar is $200 \mu \mathrm{m}$. (D) The inhibition rate of cell migration of DOX, Se@DOX, and RGDfC-Se@DOX was quantitatively analyzed. RGDfC-Se@DOX, selenium nanoparticles conjugated with RGDfC and DOX; Se@DOX, selenium nanoparticles conjugated with DOX.

Abbreviations: DOX, doxorubicin; RGDfC, Arg-Gly-Asp-D-Phe-Cys.

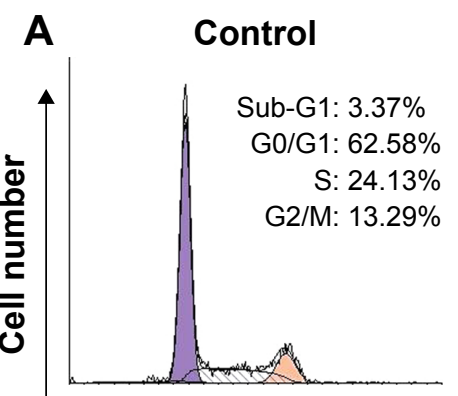

DOX

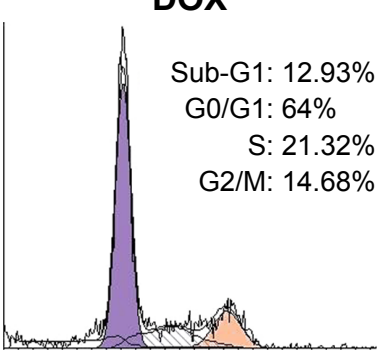

Se@DOX

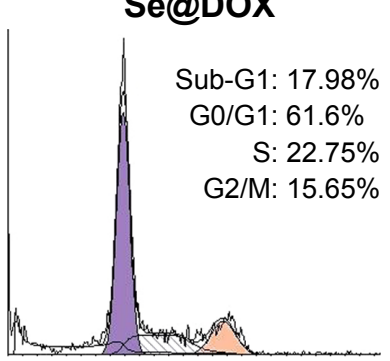

RGDfC-Se@DOX

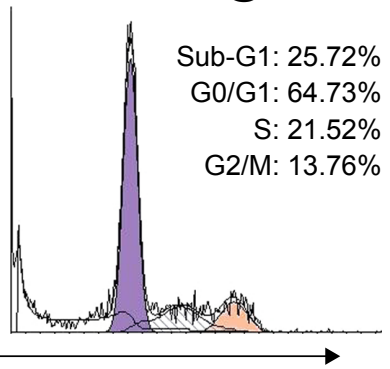

DNA content

B Control

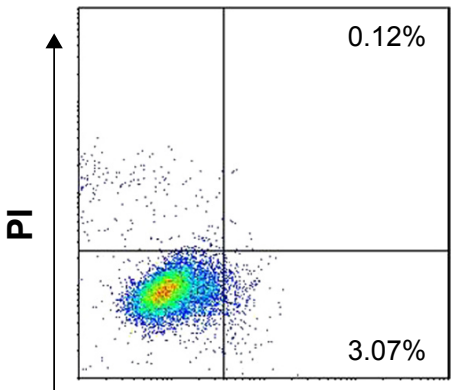

DOX

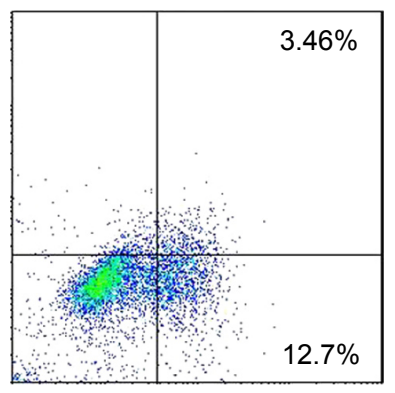

Se@DOX

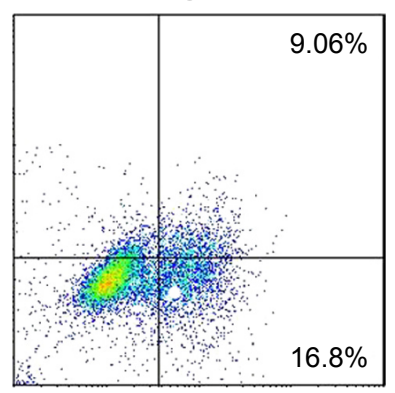

RGDfC-Se@DOX

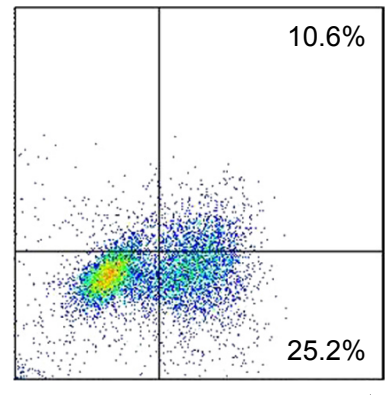

Annexin V-FITC

Figure 7 (A) Representative flow cytometry histograms of cell cycle analysis of A549 cells after incubation with various formulations of DOX for 24 h. (B) The percentages of apoptotic A549 cells after incubation with various formulations of DOX for 24 h. RGDfC-Se@DOX, selenium nanoparticles conjugated with RGDfC and DOX; Se@ DOX, selenium nanoparticles conjugated with DOX.

Abbreviations: DOX, doxorubicin; FITC, fluorescein isothiocyanate; PI, propidium iodine; RGDfC, Arg-Gly-Asp-D-Phe-Cys. 
A

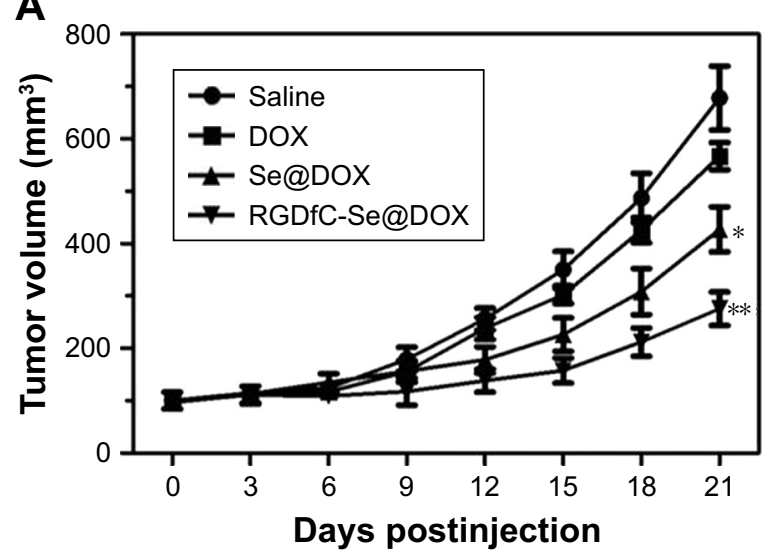

C

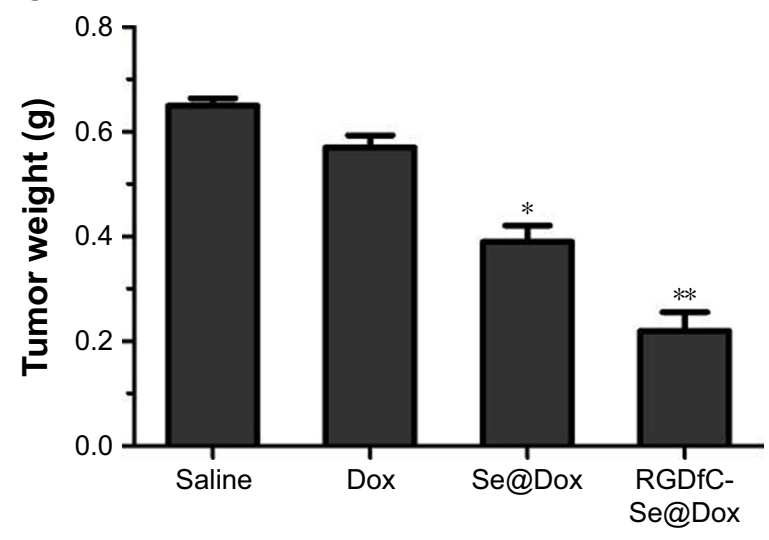

B
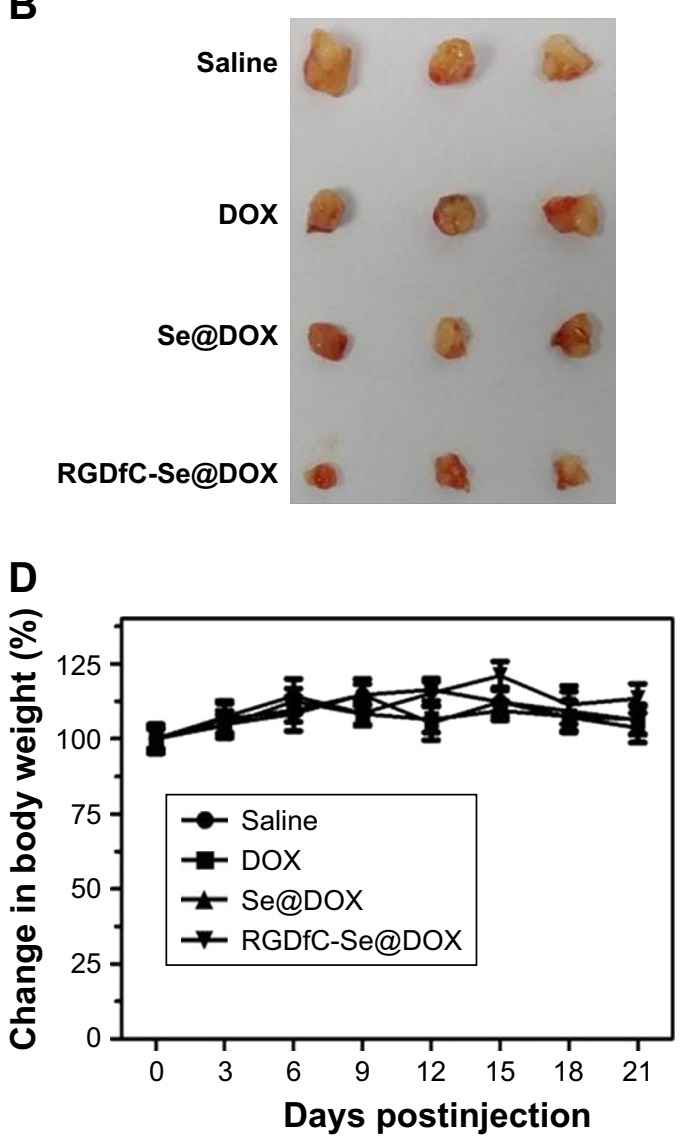

Figure 8 (A) Tumor growth curve of the xenograft nude mice bearing A549 cells after intravenous administration of saline and various formulations of DOX. (B) Morphology of all tumors stripped from the mice. (C) The tumor weight analysis of mice after 21 days of treatment. (D) The body weight change in mice during the treatment. RGDfC-Se@DOX, selenium nanoparticles conjugated with RGDfC and DOX; Se@DOX, selenium nanoparticles conjugated with DOX.

Abbreviations: DOX, doxorubicin; RGDfC, Arg-Gly-Asp-D-Phe-Cys.

rates $(35.8 \%)$ compared with the cells treated with DOX (16.16\%) or Se@DOX (25.86\%) at the equivalent DOX dose. These results indicated that RGDfC-Se@DOX could enhance the anticancer activity of DOX to induce A549 cells' apoptosis by loading the DOX onto the active tumor-targeting carrier RGDfC-SeNPs.

\section{In vivo antitumor efficacy}

The A549 tumor xenografts were used to assess the antitumor efficacy of RGDfC-Se@DOX. ${ }^{34}$ The mice were randomly assigned into four groups and then intravenously injected with RGDfC-Se@DOX, Se@DOX, free DOX, and saline, respectively. The tumor volumes and body weights of mice were tested every other day up to 21 days. As shown in Figure $8 \mathrm{~A}$, compared to the saline-treated control group, RGDfC-Se@DOX treatment obviously suppresses tumor growth during the treatment time. Moreover, RGDfC-Se@ DOX was more effective in suppressing tumor growth in comparison with free DOX or Se@DOX at the same dose of DOX, proving excellent antitumor efficacy of RGDfC-Se@
DOX. As shown in Figure $8 \mathrm{~B}$ and $\mathrm{C}$, tumor image and weight of RGDfC-Se@DOX-treated mice further verified the significant antitumor activity of RGDfC-Se@DOX. In addition, the body weight of mice kept relatively stable during treatment period, indicating that RGDfC-Se@DOX had no obvious side effects at the tested dose (Figure 8D). The in vivo antitumor efficacy showed that tumor-targeted delivery system RGDfC-Se@DOX exhibited a great potential for lung carcinoma therapy.

\section{Conclusion}

A novel active tumor-targeting SeNP RGDfC-Se@DOX was successfully synthesized to effectively deliver DOX to treat NSCLC. The RGDfC-Se@DOX showed excellent cellular uptake in NSCLC A549 cells and resulted in significant anticancer efficacy. The RGDfC-Se@DOX was more superior to inhibit A549 cell proliferation and migration/invasion and to induce A549 cells' apoptosis in vitro in comparison with free DOX and Se@DOX. Furthermore, RGDfC-Se@DOX exhibited stronger antitumor efficacy in vivo compared to 
free DOX and Se@DOX. Taken together, the tumor-targeting delivery system RGDfC-Se@DOX holds huge potential for NSCLC therapy.

\section{Acknowledgments}

This work was supported by the China Postdoctoral Science Foundation (No 2017M612632), the Science and Technology Planning Project of Guangdong Province (No 2014A020212024), the Science and Technology Planning Project of Guangdong Province (No 2015A020211002), and the Technology Planning Project of Guangzhou City (No 201607010120).

\section{Disclosure}

The authors report no conflicts of interest in this work.

\section{References}

1. Wan X, Min Y, Bludau H, et al. Drug combination synergy in wormlike polymeric micelles improves treatment outcome for small cell and non-small cell lung cancer. ACS Nano. 2018;12(3):2426-2439.

2. Yuan ZQ, Chen WL, You BG, et al. Multifunctional nanoparticles co-delivering EZH2 siRNA and etoposide for synergistic therapy of orthotopic non-small-cell lung tumor. J Control Release. 2017;268: 198-211.

3. Xiong Y, Zhao Y, Miao L, Lin CM, Huang L. Co-delivery of polymeric metformin and cisplatin by self-assembled core-membrane nanoparticles to treat non-small cell lung cancer. J Control Release. 2016; 244:63-73.

4. Au KM, Satterlee A, Min Y, et al. Folate-targeted pH-responsive calcium zoledronate nanoscale metal-organic frameworks: turning a bone antiresorptive agent into an anticancer therapeutic. Biomaterials. 2016;82:178-193.

5. Tartarone A, Rossi E, Lerose R, et al. Possible applications of circulating tumor cells in patients with non small cell lung cancer. Lung Cancer. 2017;107:59-64.

6. Sun H, Cao D, Wu H, Liu H, Ke X, Ci T. Development of low molecular weight heparin based nanoparticles for metastatic breast cancer therapy. Int J Biol Macromol. 2018;112:343-355.

7. Wang Z, He Q, Zhao W, Luo J, Gao W. Tumor-homing, pH- and ultrasound-responsive polypeptide-doxorubicin nanoconjugates overcome doxorubicin resistance in cancer therapy. $J$ Control Release. 2017;264:66-75.

8. Heger Z, Polanska H, Krizkova S, et al. Co-delivery of VP-16 and Bcl-2-targeted antisense on PEG-grafted oMWCNTs for synergistic in vitro anti-cancer effects in non-small and small cell lung cancer. Colloids Surf B Biointerfaces. 2017;150:131-140.

9. Xia F, Hou W, Zhang C, et al. pH-responsive gold nanoclusters-based nanoprobes for lung cancer targeted near-infrared fluorescence imaging and chemo-photodynamic therapy. Acta Biomater. 2018;68:308-319.

10. Mazumdar S, Italiya KS, Sharma S, Chitkara D, Mittal A. Effective cellular internalization, cell cycle arrest and improved pharmacokinetics of Tamoxifen by cholesterol based lipopolymeric nanoparticles. Int $J$ Pharm. 2018;543(1-2):96-106.

11. Sd J, Nam G-H, Kwak G, Yang Y, Kwon IC. Harnessing designed nanoparticles: current strategies and future perspectives in cancer immunotherapy. Nano Today. 2017;17:23-37.

12. Wuttke S, Lismont M, Escudero A, Rungtaweevoranit B, Parak WJ. Positioning metal-organic framework nanoparticles within the context of drug delivery - a comparison with mesoporous silica nanoparticles and dendrimers. Biomaterials. 2017;123:172-183.
13. Fang Y, Yang W, Cheng L, Meng F, Zhang J, Zhong Z. EGFR-targeted multifunctional polymersomal doxorubicin induces selective and potent suppression of orthotopic human liver cancer in vivo. Acta Biomater. 2017;64:323-333.

14. Zhou Y, Quan G, Wu Q, et al. Mesoporous silica nanoparticles for drug and gene delivery. Acta Pharm Sin B. 2018;8(2):165-177.

15. Xia Y, You P, Xu F, Liu J, Xing F. Novel Functionalized Selenium Nanoparticles for Enhanced Anti-Hepatocarcinoma Activity In vitro. Nanoscale Res Lett. 2015;10(1):349.

16. Xia Y, Lin Z, Li Y, et al. Targeted delivery of siRNA using RGDfCconjugated functionalized selenium nanoparticles for anticancer therapy. J Mater Chem B. 2017;5(33):6941-6952.

17. Li Y, Lin Z, Zhao M, et al. Multifunctional selenium nanoparticles as carriers of HSP70 siRNA to induce apoptosis of HepG2 cells. Int J Nanomedicine. 2016;11:3065-3076.

18. Li Y, Guo M, Lin Z, et al. Polyethylenimine-functionalized silver nanoparticle-based co-delivery of paclitaxel to induce HepG2 cell apoptosis. Int J Nanomedicine. 2016;11:6693-6702.

19. Li Y, Lin Z, Guo M, et al. Inhibitory activity of selenium nanoparticles functionalized with oseltamivir on H1N1 influenza virus. Int J Nanomedicine. 2017;12:5733-5743.

20. Xia Y, Wang C, Xu T, et al. Targeted delivery of HES5-siRNA with novel polypeptide-modified nanoparticles for hepatocellular carcinoma therapy. RSC Adv. 2018;8(4):1917-1926.

21. Xia Y, Xu T, Wang C, et al. Novel functionalized nanoparticles for tumor-targeting co-delivery of doxorubicin and siRNA to enhance cancer therapy. Int J Nanomedicine. 2018;13:143-159.

22. Xia Y, Guo M, Xu T, et al. siRNA-loaded selenium nanoparticle modified with hyaluronic acid for enhanced hepatocellular carcinoma therapy. Int J Nanomedicine. 2018;13:1539-1552.

23. Luesakul U, Puthong S, Neamati N, Muangsin N. pH-responsive selenium nanoparticles stabilized by folate-chitosan delivering doxorubicin for overcoming drug-resistant cancer cells. Carbohydr Polym. 2018;181:841-850.

24. Li W, Yi X, Liu X, Zhang Z, Fu Y, Gong T. Hyaluronic acid ionpairing nanoparticles for targeted tumor therapy. J Control Release. 2016;225:170-182.

25. Zhang L, Li G, Gao M, et al. RGD-peptide conjugated inulin-ibuprofen nanoparticles for targeted delivery of Epirubicin. Colloids Surf B Biointerfaces. 2016;144:81-89.

26. Wang Y, Wang X, Deng F, et al. The effect of linkers on the self-assembling and anti-tumor efficacy of disulfide-linked doxorubicin drug-drug conjugate nanoparticles. J Control Release. 2018;279:136-146.

27. Fang Z, Sun Y, Xiao H, et al. Targeted osteosarcoma chemotherapy using RGD peptide-installed doxorubicin-loaded biodegradable polymeric micelle. Biomed Pharmacother. 2017;85:160-168.

28. Li Y, Lin Z, Guo M, et al. Inhibition of H1N1 influenza virus-induced apoptosis by functionalized selenium nanoparticles with amantadine through ROS-mediated AKT signaling pathways. Int J Nanomedicine. 2018;13:2005-2016.

29. Xia Y, Chen Q, Qin X, Sun D, Zhang J, Liu J. Studies of ruthenium(ii)2,2'-bisimidazole complexes on binding to G-quadruplex DNA and inducing apoptosis in HeLa cells. New J Chem . 2013;37(11):3706-3715.

30. Zhang Q, Huang R, Tang Q, et al. Leucine-rich alpha-2-glycoprotein-1 is up-regulated in colorectal cancer and is a tumor promoter. Onco Targets Ther. 2018;11:2745-2752.

31. Zhang T, Liu W, Meng W, et al. Downregulation of miR-542-3p promotes cancer metastasis through activating TGF- $\beta / \mathrm{Smad}$ signaling in hepatocellular carcinoma. Onco Targets Ther. 2018;11:1929-1939.

32. Benfer M, Kissel T. Cellular uptake mechanism and knockdown activity of siRNA-loaded biodegradable DEAPA-PVA-g-PLGA nanoparticles. Eur J Pharm Biopharm. 2012;80(2):247-256.

33. Xia Y, Zhao M, Chen Y, et al. Folate-targeted selenium nanoparticles deliver therapeutic siRNA to improve hepatocellular carcinoma therapy. RSC Adv. 2018;8(46):25932-25940.

34. Tran TT, Tran PH, Amin HH, Lee BJ. Biodistribution and in vivo performance of fattigation-platform theranostic nanoparticles. Mater Sci Eng C Mater Biol Appl. 2017;79:671-678. 


\section{Supplementary materials}

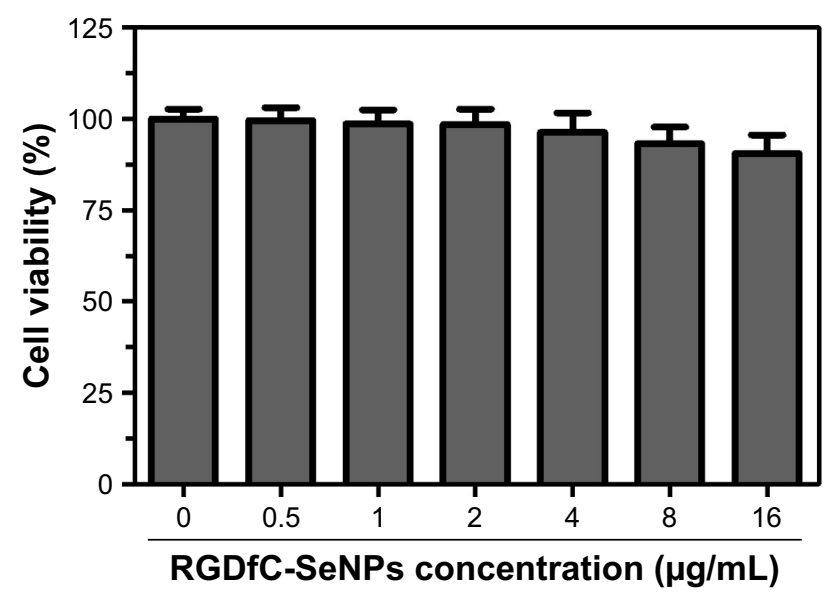

Figure SI In vitro cytotoxicity of RGDfC-SeNPs against A549 cells.

Note: RGDfC-SeNPs, SeNPs conjugated with RGDfC.

Abbreviations: RGDfC, Arg-Gly-Asp-D-Phe-Cys; SeNPs, selenium nanoparticles.

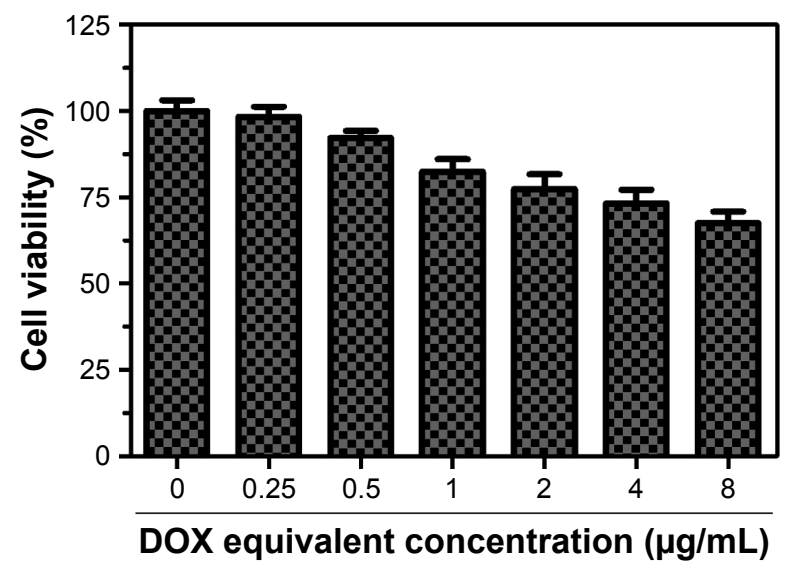

Figure S2 In vitro cytotoxicity of RGDfC-Se@DOX against HUVEC.

Note: RGDfC-Se@DOX, selenium nanoparticles conjugated with RGDfC and DOX.

Abbreviations: DOX, doxorubicin; HUVEC, human umbilical vein endothelial cells; RGDfC, Arg-Gly-Asp-D-Phe-Cys.

\section{Publish your work in this journal}

The International Journal of Nanomedicine is an international, peerreviewed journal focusing on the application of nanotechnology in diagnostics, therapeutics, and drug delivery systems throughout the biomedical field. This journal is indexed on PubMed Central, MedLine, CAS, SciSearch $®$, Current Contents $\AA /$ Clinical Medicine,
Journal Citation Reports/Science Edition, EMBase, Scopus and the Elsevier Bibliographic databases. The manuscript management system is completely online and includes a very quick and fair peer-review system, which is all easy to use. Visit http://www.dovepress.com/ testimonials.php to read real quotes from published authors. 